\title{
Characteristics of the emissive prosody of children with autism spectrum disorder
}

\author{
Patrícia Aparecida Zuanetti ${ }^{(1)}$ \\ Kelly da Silva(2) \\ Ângela Cristina Pontes-Fernandes ${ }^{(3)}$ \\ Rodrigo Dornelas ${ }^{(2)}$ \\ Marisa Tomoe Hebihara Fukuda(4)
}

(1) Hospital das Clínicas da Faculdade de Medicina de Ribeirão Preto da Universidade de São Paulo - HCFMRPUSP, Ribeirão Preto, São Paulo, Brasil.

(2) Universidade Federal de Sergipe - UFS, Campus Prof. Antônio Garcia Filho, Lagarto, Sergipe, Brasil.

(3) Universidade Paulista, Ribeirão Preto, São Paulo, Brasil.

(4) Faculdade de Medicina de Ribeirão Preto da Universidade de São Paulo FMRP-USP, Ribeirão Preto, São Paulo, Brasil.

Research carried out at Faculdade de Medicina de Ribeirão Preto da Universidade de São Paulo - FMRP-USP, Ribeirão Preto, São Paulo, Brasil.

Research support source: Fundação de Amparo ao Ensino, Pesquisa e Assistência do HCRP - USP (FAEPA)

Conflict of interests: Nonexistent

\section{(c) (i)}

Received on: February 8, 2018

Approved on: September 11, 2018

Corresponding address:

Patrícia Aparecida Zuanetti

HCRP - USP/CEOF - Centro Especializado

de Otorrinolaringologia e Fonoaudiologia

Avenida Bandeirantes, 3900

CEP: 14048-900 - Ribeirão Preto, São

Paulo, Brasil

E-mail: pati_zua@yahoo.com.br

\section{ABSTRACT}

Objective: to analyze the prosodic aspects of speech in children with Autism Spectrum Disorder.

Methods: 21 children aged 3 to 6 years participated and were divided into three groups: Group 1 - children with Autism Spectrum Disorder; Group 2 - children with language delay; Group 3 - children with typical language development. Three judges performed a subjective evaluation of the emissive prosody (characterization of pitch, loudness, sentence stress, stressed syllable and others) of the children during interaction time (video recording and use of specific protocols). The equality of proportions test was applied for group comparison $(\alpha=0.01)$.

Results: the prevalence of a history of delay for speaking the first words was high in Groups 1 and 2. Regarding the prosodic characteristics, all Group 1 children had difficulties in the use of the stressed syllable, and some in the accentuation of the sentence. On the other hand, no Group 3 children showed these changes and few Group 2 children presented excessively stressed syllable.

Conclusion: inappropriate stressing of words seems to be one of the features in the differential diagnosis of verbal children with Autism Spectrum Disorder.

Keywords: Child Language; Language Disorders; Autistic Disorder; Speech Perception; Communication 


\section{INTRODUCTION}

Autism Spectrum Disorder (ASD) is a nerodevelopmental disorder characterized by clinically significant and persistent communication and social interaction deficits, as well as limited and repetitive interest, behavior and activity patterns ${ }^{1}$. Communication deficits are variable and appear early ${ }^{2}$, ranging from absence of verbality to more specific linguistic difficulties.

The assessment of communication/language in this population should include nonverbal communication, non-literal language, suprasegmental features of speech, coherence questions, content and contingency of conversation ${ }^{3}$. In addition to language and communication, speech should also be evaluated since difficulties with articulation, voice and fluency are also observed in this population ${ }^{4}$.

One of the major challenges for an early diagnosis of children with ASD is the asystematic presence of a clear delay of the development of speech/oral language, so that some of these children may obtain a score appropriate for their age range on language development scales or standardized tests, a fact that impairs a medical diagnosis 5 . For this reason, several international researchers have started to investigate the suprasegmental features of speech in children/ adolescents with ASD in order to identify other linguistic changes that might indicate whether a verbal child has ASD $^{4-12}$.

The suprasegmental features of speech are elements that characterize larger units than phonemic features and are generally known as prosodic elements or prosody ${ }^{13}$. Prosodic traits are part of the phonologic aspect of speech; however, in contrast to phonemes, they cannot be analyzed on the basis of sound articulation since prosodic features "do not have a shape"14. Prosodic features are used to add meaning to the speech of a subject without the need of being expressed in words, and they may have a structural function (for example, interrogative/affirmative phrases) or an interpretative function (characterization of the speaker or of personal interpretations) ${ }^{13}$.

Prosody refers to pause (permits the speaker to breathe during speach, but at opportune times), intonation (emphasis or stress on a syllable within a word with variable vocal frequency), tessitura (variation of pitch), speech duration and velocity (related to the physical effects of speech production), vocal intensity/ frequency (appropriate for the context and the necessities of the speaker), and vocal quality ${ }^{13,14}$.
Prosodic changes are interpreted by the listener as negative perceptions that generate a significant interpersonal barrier between the communicating pairs $^{7,10}$. A better understanding of prosodic changes in children with a diagnosis of ASD permits us to find an additional variable that will help with the differential diagnosis of verbal children who may have ASD or another diagnosis. In addition, by diagnosing these possible changes, it will be possible to minimize losses and to contribute to a better patient adaptation in an environment in which interaction and commnication are of the utmost importance. On this basis, the objective of the present study was to analyze the emissive prosody of children with a diagnosis of Autistic Spectrum Disorder.

\section{METHODS}

This was a retrospective study approved by the Research Ethics Committee of Faculdade de Medicina de Ribeirão Preto, Universidade de São Paulo (no 7252/2016).

The study was conducted on 21 children (mean age: 4.6 years; standard deviation: 0.9 ), 15 boys and 6 girls, divided into three groups as follows:

- Group 1 (G1): children with a diagnosis of ASD

- Group 2 (G2): children with a diagnosis of delayed language development

- Group 3 (G3): children with appropriate linguistic development.

The children of the three groups were matched for sex and age.

Inclusion criteria were: children aged 3 to 6 years with borderline or medium cognitive capacity as determined in a previous psychological evaluation, and who at least emitted simple sentences. Exclusion criteria were: children with hearing deficiency, children submitted to speech therapy or with a diagnosis of syndromes.

G1 consisted of children whose diagnosis of ASD was confirmed only after evaluation/discussion by a multiprofessional team consisting of a speechhearing therapist, psychologist, occupational therapist, neurologist or child psychiatrist. G2 children were supposed to be under medical monitoring by a neuropediatrician or a team consisting of a pediatrician and a psychologist and to have a speech-hearing diagnosis of delayed development of oral language. G3 children had appropriate linguistic development.

The patients of all three groups were recruited from specific outpatient clinics of a tertiary hospital. 


\section{Materials and procedures for data collection}

The authors first analyzed the reports of evaluation of childhood language of children assessed in a Speech Therapy Service during the period from January 2015 to May 2017. These language evaluations were part of multiprofessional assessment by neurology, psychiatry or pediatrics teams. Data regarding diagnosis and medical monitoring, medical history and the results of other evaluations (psychological and auditory) were obtained from the medical records of these patients. The children to be assigned to G1, G2 and G3 were selected after analysis of the language evaluation report and of the medical records. G2 and G3 children were matched for age and sex to G1 children on a 1:1 basis.

Speech samples of the selected children were analyzed in a second stage. These samples were parts of spontaneous conversations recorded with a Sony Action video camera during ludic acivities lasting approximately 10 minutes. The speech samples were analyzed and judged by three raters. All raters were professional speech therapists with specialization in the area of language or voice who were unaware of the group to which each child belonged when analyzing the videos. It should be pointed out that the raters were submitted to a calibration process during which some voices that were not part of the investigation were presented and discussed after individual filling of the instrument. It was only after this process that the voices were presented and evaluated. After voice analysis, the Fleiss' Kappa statistical test was applied. The index of this test ranges from 0 (little concordance) to 1 (perfect concordance), with intermediate classifications as suggested by Landis and Koch ${ }^{15}$.

The protocols and variables analyzed by the raters are described in the items below.

\section{- Vocal evaluation: loudness and pitch}

The authors used part II of the Prosodic Traits of the Vocal Profile Analysis insrument by John Laver (1980), translated and adapted to Portuguese by Camargo and Madureira ${ }^{16}$. The objective of this instrument is to characterize the habitual pitch, as well as the loudmness habitually used by the subjects. Pitch and loudness were characterized using a scale from 1 (less significant) to 3 (more significant) subdivided into neutral and non-neutral scores regarding the alterations. For statistical analysis, the number of proportion of children classified with non-neutral loudness or pitch was compared between groups. We also compared the number of children with "more significant characteristics" (for example, a score of 2 or 3 for medium/high pitch, i.e., a more acute voice, or a score of 2 or 3 for loudness with reduced extension, i.e., little variation in loudness during speech).

\section{- Assessment of intonation and use of the stressed syllable}

The objective of this item is:

- to assess the intonation, i.e., the "phrase accent" (variation in melodic level which has a primary syntactic function, i.e., tonal variation that identifies a sentence as being affirmative or interrogative or of some other type).

- To assess the use of the stressed syllable within the phrase, i.e., to determine whether there is emphasis on a given word of the phrase that infers some meaning (for exeample: "Pedro did not pay the butcher's bill" - the emphasis on the "butcher" part within the phrase suggests that Pedro paid another bill, but not that of the butcher).

- To assess the use of the accent within the word (stressed syllable) since, in the case of the Portuguese language, if this accent is used incorrectly it may modify the meaning of the word.

The instrument used here for the assessment of these parameters is an adaptation of item 24 Intonation and stressed syllable of the protocol proposed by Ortiz ${ }^{17}$. For the statistical analysis of this instrument, we considered whether or not there was a change in this parameter, whereas for qualitative analysis we described the changes more frequently detected in each group.

\section{Data analysis}

Regarding the statistical tests used, the Fleiss Kappa test was employed in order to assess the reliability of the raters and the division suggested by Landis and Koch $^{15}$ was used for classification ${ }^{15}$. Concordance between raters was classified as perfect for pitch and consistency and almost perfect for loudness and for the classifications of the stressed syllable and phrase accent.

The test of Equality of Proportions between two samples was used in order to analyze the prosodic variables described above. Since this test is used to compare two groups at a time and the present study contained three groups, the level of significance was reduced based on the " $\mathrm{a} / \mathrm{n}$ - of tests" formula, so 
that the level of significance was $1.7 \%(a=0.05 ; n=$ of groups $=3$; level of significance $=0.05 / 3=0.017$ ).

\section{RESULTS}

Several variables were analyzed for better group characterization. These variables comprised possible intercurrences during the pre-, peri- and postnatal periods, as well as neuropsychomotor and linguistic development data. Table 1 presents the number of children per group with these alterations. It can be seen that the variables showing differences between some of these groups were: delayed speech/language development (both between G1 and G2 and between G2 and G3) and sleep alterations (difference between G1 and the remaining groups).

Table 1. Characterization of the groups (medical history and comorbidities)

\begin{tabular}{|c|c|c|c|c|c|c|c|}
\hline & & G1 $\left(n^{0}\right)$ & G2 $\left(n^{0}\right)$ & G3 $\left(n^{0}\right)$ & $\begin{array}{l}\text { p-value } \\
\text { G1/G2 }\end{array}$ & $\begin{array}{l}\text { p-value } \\
\text { G1/G3 }\end{array}$ & $\begin{array}{l}\text { p-value } \\
\text { G2/G3 }\end{array}$ \\
\hline \multirow{3}{*}{$\begin{array}{l}\text { Intercurrences during the } \\
\text { prenatal period }\end{array}$} & Maternal hypertension & 2 & 0 & 1 & 0.1 & 0.5 & 0.2 \\
\hline & H1N1 Influenza & 0 & 1 & 0 & 0.2 & \# & 0.2 \\
\hline & Urinary infection & 0 & 1 & 0 & 0.2 & $\#$ & 0.2 \\
\hline Prematurity & & 1 & 3 & 1 & 0.2 & 1 & 0.2 \\
\hline Intercurrences during delivery & & 0 & 0 & 0 & $\#$ & $\#$ & $\#$ \\
\hline Prenatal intercurrences & Intraventricular hemorrhage & 0 & 1 & 0 & 0.2 & $\#$ & 0.2 \\
\hline Postnatal intercurrences & & 0 & 0 & 0 & $\#$ & $\#$ & $\#$ \\
\hline Delayed NPMD & & 2 & 2 & 0 & 1 & 0.1 & 0.1 \\
\hline $\begin{array}{l}\text { Delayed emission of first } \\
\text { words }\end{array}$ & & 5 & 7 & 1 & 0.1 & $0.03^{\star}$ & $0.001^{* *}$ \\
\hline \multirow[t]{10}{*}{ Comorbidities } & Mouth breather & 1 & 0 & 2 & 0.2 & 0.1 & 0.5 \\
\hline & Childhood obesity & 1 & 0 & 1 & 0.2 & 1 & 0.2 \\
\hline & Sleep changes & 4 & 0 & 0 & $0.01 * *$ & $0.01 * *$ & $\#$ \\
\hline & Eating compulsion & 1 & 0 & 0 & 0.2 & 0.2 & $\#$ \\
\hline & Corrected visual difficulties & 0 & 1 & 0 & 0.2 & $\#$ & 0.2 \\
\hline & Visceral leishmaniasis & 0 & 1 & 0 & 0.2 & $\#$ & 0.2 \\
\hline & Nephrotic syndrome & 0 & 1 & 1 & 0.2 & 0.2 & 1 \\
\hline & Recurrent otitis & 1 & 1 & 3 & 0.2 & 1 & 0.2 \\
\hline & Cardiac changes & 0 & 1 & 0 & 0.2 & $\#$ & 0.2 \\
\hline & Congenital cytomegalovírus & 0 & 0 & 1 & \# & 0.2 & 0.2 \\
\hline
\end{tabular}

Equality of Proportions Test - *significant difference when $\alpha=0.05$; * ${ }^{*}$ significant difference when $\alpha=0.01$;

$\mathrm{n}^{0}=$ number of children with the presence of a given variablel; NPMD $=$ Neuropsychomotor development; $\mathrm{G}=$ Group 
Table 2 describes the assessment of emissive prosody of the children under study. It can be seen that all G1 children showed changes in "intonation", "emphasis on words within the phrase" and "syllabic stress", as opposed to no G3 children and to a a minimal parcel of $\mathrm{G} 2$ children. Table 3 lists in more detail (qualitative analysis) the types of changes detected regarding the item "syllable stress and intonation". The other variables (presence of tremor and/or changes in pitch and loudness) did not differ between groups.

Table 2. Analysis of emissive prosody

\begin{tabular}{|c|c|c|c|c|c|c|}
\hline & G1 $\left(n^{0}\right)$ & G2 $\left(n^{0}\right)$ & G3 $\left(n^{0}\right)$ & $\begin{array}{l}\text { p-value } \\
\text { G1/G2 }\end{array}$ & $\begin{array}{l}\text { p-value } \\
\text { G1/G3 }\end{array}$ & $\begin{array}{c}\text { p-value } \\
\text { G2/G3 }\end{array}$ \\
\hline Intonation (affirmative, interrogative etc.) & 7 & 1 & 0 & $0.001^{* *}$ & $0.0002^{* *}$ & 0.2 \\
\hline "Stressed syllable" within the phrase (word emphasis) & 7 & 1 & 0 & $0.001^{* *}$ & $0.0002^{* *}$ & 0.2 \\
\hline Word accent (stressed syllable) & 7 & 0 & 0 & $0.001^{\star *}$ & $0.001^{* *}$ & $\#$ \\
\hline Pitch (frequency) - (neutral/non-neutral) & 1 & 1 & 3 & 1 & 0.2 & 0.2 \\
\hline $\begin{array}{l}\text { Pitch - medium high (classification } 2 \text { or } 3 \text { - more } \\
\text { significant) }\end{array}$ & 1 & 1 & 3 & 1 & 0.2 & 0.2 \\
\hline $\begin{array}{l}\text { Pitch - medium low (classification } 2 \text { or } 3 \text { - more } \\
\text { significant) }\end{array}$ & 1 & 0 & 2 & 0.2 & 0.5 & 0.1 \\
\hline $\begin{array}{l}\text { Pitch - broad extension (classification } 2 \text { or } 3 \text { - more } \\
\text { significant) }\end{array}$ & 5 & 2 & 2 & 0.1 & 0.1 & 1 \\
\hline $\begin{array}{l}\text { Pitch - reduced extension (classification } 2 \text { or } 3 \text { - more } \\
\text { significant) }\end{array}$ & 0 & 1 & 1 & 0.2 & 0.2 & 1 \\
\hline $\begin{array}{l}\text { Pitch - wide variability (classification } 2 \text { ou } 3 \text { - more } \\
\text { significant) }\end{array}$ & 5 & 2 & 2 & 0.1 & 0.1 & 1 \\
\hline Vocal tremor (neutral/non-neutral) & 0 & 0 & 0 & \# & $\#$ & \# \\
\hline Loudness (intensity) - (neutral/non-neutral) & 0 & 0 & 2 & $\#$ & 0.1 & 0.1 \\
\hline $\begin{array}{l}\text { Loudness - medium high (classification } 2 \text { or } 3 \text { - more } \\
\text { significant) }\end{array}$ & 0 & 0 & 1 & 1 & 1 & 1 \\
\hline $\begin{array}{l}\text { Loudness - medium low (classification } 2 \text { or } 3 \text { - more } \\
\text { significant) }\end{array}$ & 0 & 0 & 1 & 0.2 & 0.2 & 1 \\
\hline $\begin{array}{l}\text { Loudness - broad extension (classification } 2 \text { or } 3 \text { - more } \\
\text { significant) }\end{array}$ & 4 & 3 & 3 & 0.5 & 0.5 & 1 \\
\hline $\begin{array}{l}\text { Loudness - reduced extension (classification } 2 \text { or } 3 \text { - } \\
\text { more significant) }\end{array}$ & 1 & 0 & 1 & 0.2 & 1 & 0.2 \\
\hline $\begin{array}{l}\text { Loudness - wide variability (classification } 2 \text { or } 3 \text { - more } \\
\text { significant) }\end{array}$ & 5 & 2 & 2 & 0.1 & 0.1 & 1 \\
\hline
\end{tabular}

Equality of Proportions Test $-{ }^{*}$ significant difference when $\alpha=0.05$; ${ }^{*}$ significant difference when $\alpha=0.01$; $\mathrm{n}^{0}=$ number of children classified as having "altered performance or non-neutral classification"; $\mathrm{G}=$ Group 
Table 3. Description of the types of alterations detected in the prosodic features in which there was a difference between Group 1 and the other groups

\begin{tabular}{llccc}
\hline & & $\mathbf{G 1}\left(\mathbf{n}^{\mathbf{0}}\right)$ & $\mathbf{G 2}\left(\mathbf{n}^{\mathbf{0}}\right)$ & $\mathbf{G 3}\left(\mathbf{n}^{\mathbf{0}}\right)$ \\
\hline \multirow{2}{*}{ Intonation } & Excessive melodic variation and incorrect use of intonation & 7 & 0 & 0 \\
\multirow{3}{*}{ "Phrase accent" } & Monotony & 0 & 1 & 0 \\
& Emphasis on words of non-principal categories & 7 & 0 & 0 \\
\multirow{2}{*}{ Accent (stressed syllable) } & e.g., prepositions, articles etc.) & 5 & 0 & 0 \\
& Appropriate accentuation but with excessive intensity & 5 & 0 & 0 \\
& Sporadic inadequacy of stress on the stressed syllable & 5 & 0 & 0 \\
\hline
\end{tabular}

$\mathrm{n}^{0}=$ number of children classified according to this criterion; $\mathrm{G}=$ Group

\section{DISCUSSION}

In the present study we analyzed the prosodic characteristics shown during spontaneous conversation by children with a diagnosis of ASD, children with delayed linguistic development and children with typical linguistic development in order to determine whether the possible presence of prosodic changes during early childhood could represent a marker for the differentiation of children with ASD from children with/ without other diseases.

We first observed that a history of "delayed emission of the first words" was prevalent both among children with ASD and children with delayed language development. Thus, when this complaint is present it might indicate a simple linguistic alteration which could be corrected with an appropriate diagnostic/intervention process (for example, delayed language development) ${ }^{18}$, or might indicate something more complex such as the diagnosis of ASD itself $f^{1,19}$. In the presence of this complaint, a multidisciplinary investigation is necessary for diagnostic definition and therapeutic planning within as short a time as possible, culminating with "remission of the problem" or minimization of losses in the future of these children.

Among the comorbidities cited, the only relationship strongly detected in the present study was between ASD and sleep changes. The combination of these two diagnoses is common ${ }^{20,21}$, with sleep disorders being present in more than $80 \%$ of children with ASD, ranging from refusal to go to sleep to real disorders such as insomnia and parasomnia ${ }^{21}$.

Analysis of the prosodic aspects showed that some variables differed significantly between groups, whereas others did not. Classification of vocal quality including pitch and loudness showed no difference between groups, with children with typical language development (G3) showing more alterations. In international studies assessing these same aspects (pitch, loudness and resonance) but in another age range, i.e., adolescents/adults with ASD, some of these aspects were altered, such as hypernasality ${ }^{6,10,22}$, more acute pitch $^{8,12}$ and increased speech velocity ${ }^{22}$, with the first two being more prevalent among males. The acute frequency was expected in the age range of the present study considering that, among other factors, the size of the vocal cords and of the resonance chamber influence the production of an acute sound. However, this feature may possibly persist during adult life, as reported by Wiklund ${ }^{22}$.

An extremely important result was obtained regarding intonation, phrase stress and word stress (stressed syllable), i.e., all children with ASD showed changes in these three aspects, whereas no such changes were observed at any time in the group of children with typical language development and were observed almost at no time in the group of children with language delay. This result permits us to assume that, when a child accents incorrectly a word and is unable to emit small phrases or even words with appropriate intonation, i.e., letting it be known whether the sentence is interrogative or affirmative, that child has a strong chance of having ASD.

Regarding quality, the children with a diagnosis of ASD studied here used melodic variation in an excessive manner when emitting sentences, transmitting a sensation of superficiality to the listener or making it difficult for the listener to understand the meaning of the sentence (for instance: did the child ask for something? Is the child stating that he wants something?) and incorrectly accented the syllables, changing the stressed syllable of the word or, when 
accentuating the correct stressed syllable, they used excessive vocal intensity, transmitting a sensation of strangeness to the listener. The present finding agrees with results of international studies ${ }^{5,6}$ that assessed the prosodic changes present during early childhood and which, therefore, could be of help for the differential diagnosis of these children. The changes more frequently detected in these studies involved the difficulty in accentuating the words (stressed syllable) ${ }^{5,6}$.

Most of the studies that investigate the relationship between prosodic alterations and the dagnosis of ASD have been conducted on a more advanced age range, a fact that has permitted the use of standardized tests for the assessment of linguistic prosody both from the receptive and emissive viewpoints, in addition to the acoustic analysis of voice. The results obtained in those studies, which permit to use a wider gamut of instruments for assessment and possible analyses, support the idea that subjects with ASD show prosodic changes at several levels ${ }^{7-9,12,22}$. These subjects, by having difficulty in perceiving/interpreting the prosodic characteristics of their interlocutor, understand in an erroneous manner the message emitted by him ${ }^{8,9}$ and consequently also have difficulties in producing sentences with appropriate intonation ${ }^{7-9,12}$, compromising the communicative process.

These altered linguistic "small details", added to other behavioral changes, represent an additional social and communication barrier for this population, interfering with the perception that others have of it. This may bring about problems along the life of these subjects, generating further social and emotional complications.

\section{CONCLUSION}

The present study demonstrated that children with a diagnosis of ASD who communicate in a verbal manner have changes in emissive prosody mainly characterized by inappropriate word accentuation (displacement of the stressed syllable and excessive stress on the stressed syllable) and phrase intonation. The present data, taken together with international literature findings, indicate that these specific changes may be considered to be markers of the differential diagnosis between children with ASD, children with delayed linguistic development, and children with typical linguistic development. In contrast, the complaint about delay for speaking the first words is not an exclusive complaint of the parents of children who are being evaluated for a diagnosis of ASD.

\section{REFERENCES}

1. Associação Americana de Psiquiatria - APA. Transtornos do neurodesenvovimento: transtorno do espectro autista. In: Associação Americana de Psiquiatria - APA. DSM 5 - Manual Diagnóstico e Estatístico de Transtornos Mentais. Porto Alegre: Ed. Artmed; 2014. Seção II, p. 50-9.

2. Landa R. Early communication development and intervention for children with autism. Ment Retard Dev Disabil Res Rev. 2007;13(1):16-25. DOI: https:// doi.org/10.1002/mrdd.20134.

3. Klin A. Asperger syndrome: an update. Rev Bras Psiquiatr. 2003;25(2):103-9. DOI: http://dx.doi. org/10.1590/S1516-44462003000200011.

4. Scott KS. Dysfluency in autism spectrum disorders. Procedia Soc Behav Sci. 2015;193:239-45. DOI: https://doi.org/10.1016/j.sbspro.2015.03.266.

5. McAlpine A, Plexico LW, Plumb AM, Cleary J. Prosody in young verbal children with autism spectrum disorder. Contemp Issues Commun Sci Disord. 2014:41:120-32. DOI: https://doi. org/1092-5171/14/4101-0120.

6. Shriberg LD, Paul R, McSweeny JL, Klin A, Cohen DJ, Volkmar FR. Speech and prosody characteristics of adolescents and adults with high-functioning autism and asperger syndrome. $J$ Speech Lang Hear Res. 2001;44(5):1097-115. DOI: https://doi.org/10.1044/1092-4388(2001/087)

7. Lacheret A, Normand MT, Boushaba S. Prosodic disturbances in autistic children speaking french. Speech Prosody. 2008. Acessado em outubro de 2017: http://www.academia.edu/4298789/ Prosodic_disturbances_in_autistic_children_ speaking_French.

8. Diehl JJ, Paul R. Acoustic and perceptual measurements of prosody production on the profiling elements of prosodic systems in children by children with autism spectrum disorders. Appl. Psycholinguist. 2013;34(1):135-61. DOI: https://doi. org/10.1017/S0142716411000646.

9. Paul R, Augustyn A, Klin A, Volkmar FR. Perception and production of prosody by speakers with autism spectrum disorders. J Autism Dev Disord. 2005;35(2):205-20. DOI: https://doi.org/10.1007/ s10803-004-1999-1.

10. Smerbeck AM. Nasal voice in boys with high-functioning autism spectrum disorder. Res. Autism Spectr. Disord. 2015:17:116-25. DOI: https://doi.org/10.1016/j.rasd.2015.06.009. 
11. Nash R, Arciuli J. Prosodic awareness is related to reading ability in children with autism spectrum disorders. J. Res. Read. 2016;39(1):72-87. DOI: https://doi.org/10.1111/1467-9817.12033.

12. Chan KKL, To CKS. Do individuals with high-functioning autism who speak a tone language show intonation deficits? J Autism Dev Disord. 2016;46(5):1784-92. DOI: https://doi.org/10.1007/ s10803-016-2709-5.

13. Cagliari LC. Prosódia: algumas funções dos suprassegmentos. Cad. Est. Ling. 1992;(23):137-51.

14. Lopes E. Fonética e fonologia. In: Lopes E. (org). Fundamentos da linguística contemporânea. São Paulo: Ed. Cultrix; 1995. p. 97-146.

15. Landis JR, Koch GG. The measurement of observer agreement for categorical data. Biometrics. 1977;33(1):159-74.

16. Camargo Z, Madureira S. Voice quality analysis from a phonetic perspective: voice profile analysis schemeprofile for brazilian portuguese (BP-VPAS). In: Fourth Conference on Speech Prosody Abstract book and Proceedings; Campinas. 2008. p.57-60.
17. Ortiz AZ. Avaliação das disartrias. In: Ortiz KZ. (org). Distúrbios neurológicos adquiridos. Barueri: Ed Manole; 2010. p. 92.

18. Zorzi JL. Aspectos básicos para compreensão, diagnóstico e prevenção dos distúrbios de linguagem na infância. Rev. CEFAC. 2000;2(1):11-5.

19. Zanon RB, Backes B, Bosa CA. Identificação dos primeiros sintomas do autismo pelos pais. Psic.: Teor. e Pesq. 2014;30(1):25-33. DOI: http://dx.doi. org/10.1590/S0102-37722014000100004.

20. Devnani PA, Hegde AU. Autism and sleep disorders. J Pediatr Neurosci. 2015;10(4):304-7. DOI: http://dx.doi.org/10.4103/1817-1745.174438.

21. Liu X, Hubbard JA, Fabes RA, Adam JB. Sleep disturbances and correlates of children with autism spectrum disorders. Child Psychiatry Hum Dev. 2006;37(2):179-91. DOI: http://dx.doi.org/10.1007/ s10578-006-0028-3.

22. Wiklund M. Interactional challenges in conversations with autistic preadolescents: The role of prosody and non-verbal communication in other-initiated repairs. J. Pragmat. 2016;94:76-97. DOI: https://doi.org/10.1016/j.pragma.2016.01.008. 